\title{
Research on the Coordination of Environmental Pollution and Industrial Structure Upgrading from the Coupling Perspective
}

\author{
Wenjing Zhang ${ }^{1}$ \\ ${ }^{1}$ School of Management, Tianjin University of Technology, Tianjin, China
}

\begin{abstract}
Economic transformation and industrial structure upgrading are critical to achieving environmentally sustainable development. Based on the inaccurate data analysis of the VEC model and the logarithmic average exponential decomposition method, this paper proposes an analysis of the two-way coupling relationship between natural gas energy consumption and environmental pollution. Through the identification of conceptual models of the re-linking and decoupling relationship between energy consumption and environmental pollution, two-dimensional endogenous variables and exogenous variables are determined. The research results show that both energy consumption and $\mathrm{CO} 2$ emissions are on the actual data statistical line, with an error of 0 . At the end, the article puts forward policy recommendations to promote the coordinated development of urban agglomeration environmental pollution and industrial structure upgrading.
\end{abstract}

\section{Introduction}

On October 26, 2020, the Fifth Plenary Session of the 19th Central Committee clearly emphasized the need to achieve the new requirements of the "ecological civilization construction to achieve new progress" and "economic development to achieve new results", which means that domestic industrial development must take into account economic transformation and environmental sustainability. Dual goals. Reasonable environmental regulations can not only achieve the goal of environmental improvement, but also achieve green economic development by optimizing the industrial structure ${ }^{[1]}$. It can be seen that attaching importance to environmental protection and upgrading the industrial structure has very important practical significance for realizing environmental protection and economic development. The use of energy, such as natural gas in the process of drilling, mining, gathering and transportation, and purification, will produce various tail gas and waste residues, which cause a lot of pollution to the environment and endanger human health ${ }^{[2]}$. In terms of drilling wastewater pollution, the treatment has achieved initial results, and the pollution area and scope have been initially controlled. However, large-scale and in-depth treatment cannot effectively control the pollution caused by desulfurization tail gas, gas field water, and operation wastewater. It is still difficult to solve the problem deeply troubles the country and the people ${ }^{[3]}$.

Scholars have conducted a lot of related paradigm studies on carbon emissions. Zhou Bin et al. ${ }^{[4]}$ believe that "by integrating the low-carbon-circular economy coupling development model into the planning development ideas, main goals, spatial layout, and key industries, the spatial distribution reflects the circulation of regional resources; in key industries Zhongjun runs through the low-carboncircular economy concept design industry chain, especially the vein industry designed to lay the foundation for the smooth implementation of the circular economy industrial cluster." Yang Zhicheng ${ }^{[5]}$ discussed in depth the changes in the total amount of carbon "sources" and economic development. The relationship between carbon emission structure and economic structure. It is believed that the distribution of carbon emissions reflects social and economic factors such as the total economic volume, structure, efficiency, and population of the country and region, and it is recommended to strengthen the research and monitoring of the dynamic changes of carbon "source/sink". Wang Mingyue et al. ${ }^{[6]}$ believe that "lowcarbon circular economy is an organic combination of low-carbon economic model and circular economy model. It is a 'two-dimensional' concept and a strong coupling model. Low-carbon circular economy has resource efficiency and carbon emissions. It is the driving force, government guidance is a reliable guarantee for strong coupling, remanufacturing is a new bright spot, industrial clusters and symbiosis support carriers in the park, and the necessary conditions for the industrial symbiosis coupling structure." It can be seen that the carbon circular economy as an energy sector reduces CO2. Discharge methods have attracted the attention of experts and institutions. According to previous studies, the energy consumption of natural gas is determined through the use of the VEC model; the logarithmic average index decomposition method is used to analyze the energy consumption of natural gas based on environmental pollution driving factors ${ }^{[7]}$. However, it is extremely rare to analyze the relationship between the two under the perspective of coupling. Therefore, discuss the coupling relationship between natural gas energy consumption and 
environmental pollution, prove the potential of energy saving and emission reduction, for reference by the natural gas service industry, and provide valuable theoretical basis and data support for the low-carbon development of the natural gas service industry.

\section{Quantitative analysis of the coupling relationship between energy consumption and environmental pollution}

\subsection{Analysis of the relationship between energy consumption and environmental pollution}

Energy promotes economic development, but it also causes serious pollution to the human living environment ${ }^{[8]}$. The current energy production and use of natural gas produce a large amount of harmful gases, waste liquids and wastes, which not only pollute the atmosphere, rivers, and land, but even destroy ecological balance, food safety and other issues, and seriously threaten human health and the living environment. Energy consumption and environmental pollution problems refer to the environmental effects produced by human beings in the process of mining, transportation, processing, transformation and utilization of energy. How to solve the pollution of natural gas energy emissions is one of the biggest problems facing the modern environment. While energy consumption continues to improve human material life, it also deteriorates the human living environment. To achieve sustainable development, mankind must resolve this contradiction.

\subsection{Metrological analysis}

\subsubsection{Energy consumption}

According to the "General Principles of Comprehensive Energy Consumption Calculation", the comprehensive energy consumption of energy consumption mainly includes various energy consumed by the production system, auxiliary production system and auxiliary production system, energy conversion losses, and energy consumption of raw materials. Therefore, the "Statistical Yearbook" "Include selection. Therefore, the statistical limit of energy consumption is set as the entire coal-to-gas production process. The energy of the input device includes raw coal, fuel coal and other electricity consumption, and the energy of the output system includes by-products such as external air.

\subsubsection{Environmental pollution}

Environmental pollution indicators are industrial waste gas emissions in units of 100 million standard cubic meters, industrial wastewater emissions in units of 10,000 tons, and industrial waste solid emissions in units of 10,000 tons.

According to the statistical yearbook, the formula for estimating $\mathrm{CO} 2$ emissions from the carbon emissions data generated by fuel is as follows:

$$
\mathrm{MCO}_{2 j}=\text { qnet }_{j} \times 10^{-9} \times \text { ratio }_{c_{-j}} \times 10^{-3} \text { (1) }
$$

In the calculation formula, qnet ${ }_{j}$ represents the low calorific value generated by the use of natural gas; ratio $_{c_{-j}}$ represents the carbon oxidation rate. Obtain the following formula to calculate the $\mathrm{CO} 2$ emissions of province $\mathrm{i}$ in year $\mathrm{t}$ :

$$
C O_{2 i, t}=\left(\sum_{j}^{8} M_{-} C O_{2 j}\right) \times q_{i, j, t}
$$

In the calculation formula, $q_{i, j, t}$ represents the natural gas energy consumption degree of province $i$ in year $t$.

\section{Coupling model construction}

Analyze the impulse disturbance response of environmental pollution and energy consumption through the impulse response function, and obtain the following impulse response function calculation formula:

$$
x_{i}=b^{0} \mu_{j}+b^{1} \mu_{j-1}+b^{2} \mu_{j-2}
$$

In the calculation formula, $\mathrm{b}^{j}$ represents the impulse function obtained by impulsively affecting the twodimensional endogenous variables between natural gas energy consumption and environmental pollution.

Through the changes of endogenous variables in the two-way coupling model of natural gas energy consumption and environmental pollution, the importance of the contribution of different information shocks can be evaluated. According to the two-way coupling relationship between environmental pollution and energy consumption, the variance analysis is carried out, and the expression (4) of the variance decomposition is:

$$
\mathrm{RVC}=\frac{\sum_{i=0}^{\infty}\left(k^{t}\right)^{2} \mu_{j}}{\sum_{j=1}^{k}\left[\sum_{i=0}^{\infty}\left(k^{i}\right)^{2} \mu_{j}\right]}
$$

\section{Coupling relationship analysis}

Using the coupling relationship between natural gas energy consumption and environmental pollution to analyze the energy consumption decoupling elastic coefficients are all located in the first quadrant, distributed in the expansion negative decoupling zone, expansion connection zone and weak decoupling zone, most of which are located in the weak decoupling zone. The study found that the economic growth rate of the natural gas service industry during the observation period was higher than that of most years; although it did not reach the ideal strong decoupling state, the overall decoupling state was relatively good. Set 10-period impulse response, obtain the impulse response relationship between energy consumption and environmental pollution as shown in Table 1.

Table.1 Energy consumption and environmental pollution response value

\begin{tabular}{lclc}
\hline Shock & Impact & Solid & Liquid \\
period & $\begin{array}{c}\text { periodCO2Emission } \\
\text { response value }\end{array}$ & $\begin{array}{l}\text { emissions } \\
\text { Response }\end{array}$ & $\begin{array}{l}\text { discharge } \\
\text { Response }\end{array}$ \\
\hline
\end{tabular}




\begin{tabular}{cccc}
\hline & & value & value \\
\hline 1 & 0 & 0 & 0 \\
\hline 2 & -0.15 & -0.05 & 0 \\
\hline 3 & -0.22 & -0.15 & -0.05 \\
\hline 4 & -0.28 & -0.14 & 0.1 \\
\hline 5 & -0.3 & -0.13 & 0.12 \\
\hline 6 & -0.26 & -0.10 & -0.11 \\
\hline 7 & -0.23 & -0.08 & 0.09 \\
\hline 8 & -0.18 & -0.07 & 0.08 \\
\hline 9 & -0.15 & -0.05 & 0.06 \\
\hline 10 & -0.1 & -0.01 & 0.05 \\
\hline It
\end{tabular}

It can be seen from Table 1 that the energy consumption response values corresponding to $\mathrm{CO} 2$ emissions and solid emissions are all negative numbers. Therefore, the relationship between energy consumption and $\mathrm{CO} 2$ emissions and solid emissions is negative; while the relationship between liquid emissions is negative. The response value fluctuates on the zero value line, so it is concluded that the liquid discharge volume and the energy consumption volume are restricted.

\section{Coupling mechanism design}

\subsection{Data collection}

By querying the original data of China Statistical Yearbook and China Energy Statistical Yearbook from 2015 to 2020, draw the following table, and deeply explore the coupling relationship between energy consumption, $\mathrm{CO} 2$ emissions and the environment.

Table 2. Energy consumption and $\mathrm{CO} 2$ emissions statistics table

\begin{tabular}{ccc}
\hline years & $\begin{array}{c}\text { Energy } \\
\text { consumption } \\
/ 10^{11} \mathrm{KJ}\end{array}$ & $\begin{array}{c}\text { CO2 emissions } \\
/ 10^{4} \mathrm{t}\end{array}$ \\
\hline 2015 & 45978.62 & 39850.85 \\
\hline 2016 & 48745.62 & 42435.75 \\
\hline 2017 & 54112.15 & 47533.15 \\
\hline 2018 & 63584.65 & 55115.30 \\
\hline 2019 & 69551.85 & 60525.25 \\
\hline 2020 & 85120.15 & 68452.01 \\
\hline
\end{tabular}

\subsection{Result analysis}

This paper uses the VEC model, log-average exponential decomposition method and coupling model analysis, and finds that the energy consumption and $\mathrm{CO} 2$ emissions obtained using the VEC model have a large error; the energy consumption and $\mathrm{CO} 2$ emissions obtained using the log-average exponential decomposition method The emission error value is smaller than that used in the VEC model; the energy consumption and $\mathrm{CO} 2$ emission using the coupled model both reach the actual data statistical standard, and the error is 0 . Based on this, it is concluded that the coupling model analysis effect is good.

\section{Conclusions and recommendations}

This paper constructs a coupling model based on the decoupling and double hooking theory to analyze the relationship between natural gas energy consumption and environmental pollution. Through experiments, it is known that the model can accurately determine the relationship between natural gas energy consumption and environmental pollution and has a good analytical effect. Under the background of the new normal, on the basis of ensuring the quality of economic development and the continuous expansion of economic scale, reducing the growth rate of energy consumption and achieving zero growth in energy consumption is an arduous and urgent task. Based on the analysis results of this article, we plan to propose a regulation path value to reduce the growth of energy consumption from the following three points:

First, in the path of new urbanization, the government should thoroughly implement the concept of energysaving consumption, scientifically guide the population growth of the country, especially the central and western regions, and actively promote the construction of small and medium-sized cities, which will reduce the energy consumption and the impact of population growth. It is of great significance to coordinate the balance of regional development. Through the dual means of policy incentives, publicity, and training, people's traditional consumption outlook is changed.

Second, give full play to the leverage of energy prices and vigorously promote the reduction of industrial energy intensity. Continue to strengthen scientific and technological innovation, make full use of the advantages of energy price leverage, transform traditional industries, reduce the use of high-polluting energy sources such as crude oil, fuel oil, coal, gasoline, and promote emerging industries, and strive to tap and reduce energy sources in various industries, especially high-energy-consuming industries. Strengthen the potential, actively develop renewable energy and new energy, increase the proportion of renewable energy, and explore a diversified energy consumption structure.

Third, optimize the direction of industrial structure transformation, and earnestly exert the energy consumption reduction effect of industrial structure adjustment. Reduce dependence on foreign-funded enterprises, guide capital to flow to high-end industries, take extensive application of high-tech and advanced applicable technologies to transform and upgrade traditional superior industries as an important task, provide support from various aspects such as policies, funds, and talents, promote corporate restructuring and eliminate backward production capacity, to inject vitality and vitality into the transformation and upgrading of traditional industries, and promote the upgrading and transformation of industrial structure.

\section{References}

1. Yang Qian, Qin Wenjin, Liu Huajun. Does environmental regulation promote the optimization and upgrading of industrial structure? $[\mathrm{J}]$. Shanghai 
Economic Research, 2019, (6): 83-95.

2. Yang Xiaoye, Mi Yanxiang, Xu Donghai. Coupling analysis of environmental pollution and environmental governance - taking industrial waste gas emission and governance as an example $[\mathrm{J}]$. Ecological Economy, 2019, 35(1): 191-194; 205.

3. Guan Xueling, Zhou Min. Research on the Coupling Development of Urbanization and Energy Consumption $[\mathrm{J}]$. Journal of China University of Mining and Technology, 2019, 48; 231(6): 226-233.

4. Zhou Bin, Chen Xingpeng, Xue Bing, et al. Theoretical research and empirical research on the coupling development model of low carbon and circular economy [J]. Resources and Industry, 2010 (6): 19-27.

5. Yang Zhicheng. Carbon cycle and economic development [J]. Science and Technology Plaza, 2012 (6): 122-127.

6. Wang Mingyue, Zhang Qiqi, Shi Wenqiang. Research on the Connotation and Development Strategy of Low-Carbon Circular Economy [J]. Journal of Northeast Agricultural University (Social Science Edition), 2017 (5): 46-52.

7. Chen Yue, Wei Wei, Liu Feng, etc. Optimal decisionmaking of consumers in thermal-electricity market based on CES-type utility function $[\mathrm{J}]$. Automation of Electric Power Systems, 2018, 42(13): 118-126.

8. Wang Renxiang, Lu Pengfei. The coupling relationship between technological innovation, green finance and industrial policy - - Based on the analysis of my country's energy conservation and environmental protection industry $[\mathrm{J}]$. Journal of Beijing University of Posts and Telecommunications (Social Science Edition), 2019, 21(1): 30-41. 